Trauma Berufskrankh $2005 \cdot 7[$ Suppl 1]:S247-S252 DOI 10.1007/s10039-005-0999-5

Online publiziert: 29. Januar 2005

(c) Springer Medizin Verlag 2005

\title{
M. Steen
}

Klinik für Plastische und Handchirurgie, Brandverletztenzentrum, BG-Kliniken Bergmannstrost, Halle, Saale

\section{Spezielle handtherapeutische Rehabilitation}

zept entwickelt, welches unter handchirurgischer Führung noch notwendige Diagnostik, multidisziplinäre Therapieansätze und Testverfahren sowie den Übergang in die berufliche Rehabilitation vereinigt. Es ist seit 5 Jahren praktisch erprobt und hat breite Akzeptanz und Inanspruchnahme durch die BG gefunden.

In diesem Beitrag stellen wir

- die Konzeption,

- die zur Verfügung gestellten Möglichkeiten und - die Organisation

dieser speziellen Handrehabilitation vor.

Ihre Entwicklung begann als interne Veränderung der BGSW zum 01.07.2001. Zu diesem Zeitpunkt wurde eine interdisziplinäre Visite eingerichtet. Durch die Akzep$\tan z$ und Inanspruchnahme von Seiten der BG kam es zu einer stetigen Mengenentwicklung. $\mathrm{Zu}$ Beginn des Jahres 2003 waren die Kapazitäten so weit überlastet, dass sie limitierender Faktor für die weitere Entwicklung wurden. Zum 01.04.2003 wurden im Haus deshalb zusätzliche Betten zugewiesen, ab 01.10.2003 wurden 10 Hotelbetten nach Vereinbarung mit dem Landesverband Nordwestdeutschland angemietet, um die Kapazität zu erhöhen. Seit Frühsommer 2004 stehen am Bergmannstrost 24 Betten in ausgebauten 2-Zimmer-Appartments als Hotelkapazität zur Verfügung. Die komfortable Unterbringung außerhalb der Stationspflege ermöglichte zusätzlich eine Senkung der Preise bei gleichzeitiger Intensivierung des medizinischen Programms.

\section{Zielgruppen der handtherapeutischen Rehabilitation}

Von der handtherapeutischen Rehabilitation profitieren können:

- Verletzte mit komplexen Handverletzungen

- Verletzte mit gravierenden Funktionsstörungen nach schwer wiegenden Komplikationen [z. B. Infektion, CRPS (Morbus Sudeck)]

- Verletzte mit leichteren Verletzungen bis hin zu Bagatellverletzungen, bei denen das Heilverfahren durch verschiedene Faktoren prolongiert worden ist und das bisher erreichte Ergebnis keinen Abschluss ermöglicht

Bei diesen Verletztengruppen war es häufig durch das Verletzungsausmaß oder unzureichende Therapie zu objektiv schlechten Ergebnissen gekommen, wobei Gelenkeinsteifungen durch fehlende Übung eine erhebliche Rolle innehatten. Diese Verletzten profitieren von einem konzentrierten Programm.

Für die Kostenträger ist von wesentlicher Bedeutung, dass in kurzer Zeit mit konzentriertem Einsatz der Mittel meist eine wesentliche Verbesserung erreicht werden kann, welche eine berufliche Rehabilitation ermöglicht.

Durch denEinsatzentsprechender Messtechnik und der Erfahrung des Behandlerteams gelingt es zudem, Verletzte mit Aggravation, Simulation und Rentenbegehren zu identifizieren und dies in vielen Fällen so weit zu klären, dass der Patient diesen Weg nicht weiterverfolgt oder verfolgen kann. gig zu Ende zu führen, haben wir ein solches, multidisziplinär ausgerichtetes Kon- 
Auch die Abgrenzung von erreichten Beharrungszuständen ist mit der vorhandenen Messtechnik möglich und kann damit helfen, Heilverfahren auf einem erreichten Stand abzuschließen und längere, erfolglose, aber kostenträchtige Therapiebemühungen einzugrenzen.

\section{Behandlungsziele}

Sie sind die möglichst weitgehende Wiederherstellung der Handfunktion und die Wiedereingliederung in den Arbeitsplatz bzw. ausgeübten/erlernten Beruf. Dies erfordert:

- frühzeitige Entscheidung über noch notwendige diagnostische und operative Maßnahmen

- auf die Verletzungsfolgen und den beruflichen Einsatz ausgerichtetes Rehabilitationsprogramm

- bestmögliche Wiederherstellung von Funktion, Geschicklichkeit und Kraft

- Dokumentation aller Ausgangsbefunde, des Verlaufs und der Abschlussbefunde

\section{Medizinische Konzeption}

In der handtherapeutischen Rehabilitation wird die Funktionsstörung der oberen Extremität analysiert. Die bisher bekannten Unfallfolgen werden auf evtl. bisher übersehene oder unterschätzte weitere Folgen, aber auch auf ihren Unfallzusammenhang überprüft.

Einsteifungen der Gelenke, Blockierungen durch Verwachsungen am Bewegungsapparat, nervale Schäden und Störungen der Zirkulation, um nur einige Aspekte zu nennen, werden in Verbindung mit der Motivation des Verletzten, seinem Schmerzerleben und seiner Verarbeitung von Trauma und aktuellen Beschwerden eingeschätzt. Gerade die letztgenannten Faktoren sind eine häufige Ursache für verzögerte oder nicht erfolgreiche Rehabilitation.

Basis aller Maßnahmen ist eine Objektivierung der Störungen und des Behandlungsfortschritts. Die dazu notwendige Messtechnik und die damit verbundenen Untersuchungsmethoden sind im handchirurgischen Funktionslabor konzentriert, in dem die Messungen durchgeführt und dem Behandlerteam zur Verfügung gestellt werden. Auch für das Schmerzempfinden und die psychopathologische Ebene lassen sich Objektivierungen erreichen, welche die Abgrenzung unfallbezogener und unfallfremder Faktoren erleichtern und in weitere Therapieansätze führen.

Eine wesentliche Rolle spielt auch die Berücksichtigung der zukünftigen Möglichkeiten des Verletzten. Eine frühzeitige Orientierung auf die berufliche Rehabilitation und ggf. erste Schritte in der Erstellung beruflicher Belastungsprofile sind in die Ergotherapie integriert und mit der Berufshilfe von Beginn an abgestimmt. Die Einbeziehung der Orthopädiemechaniker und von Mitarbeitern eines Sanitätshauses ermöglicht, soweit erforderlich, eine gezielte Hilfsmittelversorgung zu einem frühen Zeitpunkt.

Die Einbindung von Berufshilfe und Sozialarbeit rundet das Team ab und ermöglicht eine nahtlose Überleitung handtherapeutischer Rehabilitation in berufliche Belastungserprobung (ABE) oder die Wiederaufnahme der Arbeit, Meldung beim Arbeitsamt oder Beginn von berufsfördernden Maßnahmen durch die BG.

\section{Behandlerteam}

Es trägt dem interdisziplinären Ansatz Rechnung. Es setzt sich aus folgenden Bereichen zusammen:

- Arzt der Handchirurgie

- Arzt mit dem Spezialgebiet Schmerztherapie (Anästhesiologe)

- Dipl.-Psychologe/psychologischer Psychotherapeut

- Arzt mit Schwerpunkt physikalische und rehabilitative Medizin

- Physiotherapeuten

- Ergotherapeuten

- Sporttherapeuten

- handchirurgisches Funktionslabor

- Berufshilfe

- Orthopädietechniker

- Pflegekräfte der handchirurgischen Station

Bei Bedarf werden u. a. folgende Fachgebiete eingebunden

$$
\begin{aligned}
& \text { - Unfallchirurgen } \\
& \text { - Orthopäden } \\
& \text { - Neurologen }
\end{aligned}
$$

- Radiologen

- Internisten

\section{Organisation}

Kristallisationspunkt zum Austausch aller Informationen im Behandlerteam ist eine Besprechung einmal wöchentlich, an dem alle Mitglieder des Kernteams teilnehmen. Hier werden die Fortschritte und Konzepte ausgetauscht und miteinander abgestimmt. Die Verletzten werden in diese Teamsitzungen einbezogen und erfahren damit eine Motivationsverstärkung, da erreichte Ziele und neue Vorgaben dort besprochen werden.

Alle Berufsgruppen des Teams organisieren nach diesen Absprachen ihren Behandlungsbereich. Treten neue Gesichtspunkte auf, erfolgt eine Rückkopplung mit allen anderen Behandlern. Wichtige Ergebnisse werden allen auch außerhalb der Teamsitzungen mitgeteilt. Damit kann eine hohe Effizienz bei guter Abstimmung erreicht werden.

Das Übungsprogramm ist für die Verletzten Montag bis Freitag ganztägig, am Samstag halbtags strukturiert und umfasst eine Übungszeit von täglich etwa $7 \mathrm{~h} \mathrm{zu}$ züglich der notwendigen Pausen, Essenszeiten und Wege.

Im Regelfall gehen wir von einer Dauer der handtherapeutischen Rehabilitation von 3 Wochen aus. Diese Zeit reicht in den meisten Fällen aus, eine berufliche Wiedereingliederung zu erreichen. Eine Verkürzung ist ebenso wie eine Verlängerung in $\mathrm{Ab}$ stimmung mit dem Kostenträger möglich. Entscheidend für die Dauer der Maßnahme sind das Erreichen der für den Verletzten festgelegten Ziele oder eine nachgewiesene Begrenzung des Übungsfortschritts mit Erreichen eines Beharrungszustands.

\section{Eingesetzte Therapieverfahren}

Jeder Bereich des handtherapeutischen Teams organisiert seine Behandlung in Absprache über die Teamsitzungen. Damit wird gewährleistet, dass jeder Bereich seine Möglichkeiten ausschöpft und umfangreich und koordiniert einsetzt. Die Bereiche optimieren ihre Verfahren und halten sie auf dem aktuellen Stand der wissenschaftlichen Entwicklung. Über das Behandlerteam erfolgen die interne Qua- 


\section{Zusammenfassung $\cdot$ Abstract}

litätskontrolle und der Austausch über neue Methoden und Verfahren.

Die nachfolgende Aufstellung umfasst die von den einzelnen Bereichen vorrangig angebotenen und eingesetzten Verfahren.

\section{Handchirurgie}

In ihr erfolgen die Eingangsuntersuchung und die Indikationsstellung für eine handtherapeutische Rehabilitation. Zu den weiteren Aufgaben gehören:

- Erhebung des Aufnahmebefunds

- Festlegung des Behandlungskonzepts

- Indizierung und Veranlassung aller notwendigen weiteren Diagnostik und Therapie in Abstimmung mit den anderen Bereichen

- Kontrolle des Verlaufs, Änderungen der Therapie

- Patientenführung

- Erstellung und Kontrolle der Dokumentation

- Abschlussuntersuchung und Berichterstellung, ggf. Begutachtung

- Festlegung und Durchführung evtl. diagnostischer (z. B. Arthroskopie) oder therapeutischer Eingriffe (Operationen)

\section{Schmerztherapie}

Folgende Schmerzsyndrome werden durch die Abteilung Schmerztherapie der Klinik für Anästhesiologie, Intensiv- und Notfallmedizin im Rahmen der Handtherapie behandelt:

- Nervenschmerzen nach Unfällen

- Phantom- und Stumpfschmerzen nach traumatischen Amputationen

- CRPS I und II (sympathische Reflexdystrophie, Kausalgie)

- periphere neuropathische Schmerzsyndrome

- muskuloskelettale Schmerzsyndrome (Arthrose, Muskel-Sehnen-Verletzungen)

- chronische Schmerzerkrankung (Stadium 1-3)

Dabei werden folgende schmerztherapeutische Techniken angeboten und ausgeführt:

1. Medikamenteninfusion

- Lidocaininfusion

- Procainfusion
Trauma Berufskrankh $2005 \cdot 7[$ Suppl 1]:S247-S252

DOI 10.1007/s10039-005-0999-5

(c) Springer Medizin Verlag 2005

M. Steen

\section{Spezielle handtherapeutische Rehabilitation}

\section{Zusammenfassung}

Handtherapeutische Rehabilitation ist ein Prozess, der die gesamte Kette von der suffizienten primären Versorgung bis zur beruflichen Wiedereingliederung umfasst.

Für die Qualität der direkten medizinischen Therapie, insbesondere der operativen Versorgung, sind in den vergangenen Jahrzehnten hohe Standards entwickelt worden und Spezialabteilungen entstanden. Auch die Physiotherapie und Ergotherapie haben sich der Hand als funktionellem Organ vermehrt zugewandt. In jüngster Zeit gewinnen Bemühungen der Berufsgenossenschaften an Bedeutung, auch die Qualität bei der Versorgung der "kleinen Handverletzungen", welche meist in nicht spezialisierten Einrichtungen behandelt werden,

zu verbessern. Zur Komplettierung der Rehabilitationskette haben wir ein Programm zur handtherapeutischen Rehabilitation entwickelt und seit 4 Jahren in der Praxis weiterentwickelt, welches für schwere Handverletzungen ebenso eingesetzt werden kann wie für komplizierte Verläufe, prolongierte Heilverfahren und Verletzte mit Aggravationstendenzen. Im vorliegenden Beitrag werden das Konzept und die Organisation dieser speziellen handtherapeutischen Rehabilitation vorgestellt.

\section{Schlüsselwörter}

Handtherapie · Rehabilitation .

BG-Heilverfahren

\section{Special hand therapeutic rehabilitation}

\section{Abstract}

Hand therapeutic rehabilitation is a process in which the whole sequence from sufficient primary care to return to work are included. High standards have been developed and special units created to ensure the quality of direct medical therapy, and in particular surgical care. Physiotherapy and ergotherapy have also given more recognition to the hand as a functional organ. Recently, professional associations have been succeeding in their efforts to improve the treatment of "minor" hand injuries which are not usually treated in specialist units. To com- plete the sequence of rehabilitation, we have developed a program dealing with hand therapy over 4 years of practice. This can be used for severe hand injuries as well as those with a complicated course, prolonged healing or a tendency to being aggravated. In this study, the concepts and organisation of this special hand therapeutic rehabilitation are detailed.

\section{Keywords}

Hand therapy · Rehabilitation .

Specialist organisation-treatment 
- Opioidinfusion mit Fentanyl

- balancierte Kurzzeitanalgosedierung (BAKAS)

2. regionalanästhesiologische Techniken und Blockade einzelner Nerven (hier eingegrenzt auf die obere Extremität)

- Plexus brachialis interskalenäre Blockade infraklavikulare Blockade axillare Blockade

- einzelne Armnerven rückenmarknahe Analgesie Spinalanästhesie

Periduralanästhesie epidurale, perineurale Medikamentenapplikation intrathekale Medikamentenapplikation

- Sympathikusblockaden Blockade des Ganglion cervicale superior Blockade des Ganglion stellatum i. v. regionale Sympathikusblockade

- Implantation von Rückenmarkstimulationsgeräten

- transkutane elektrische Nervenstimulation

3. Triggerpunktinfiltrationen

4. manuelle Therapie

5. schmerzmedikamentöse Einstellung der Patienten

Im Einzelfall kann auch auf Akupunktur zurückgegriffen werden.

\section{Medizinische Psychologie}

In der Handtherapie ergeben sich folgende Indikationen für eine psychotherapeutische Intervention:

- individuelle Verarbeitung des Unfalltraumas

- psychoreaktive Störungen (z. B. posttraumatische Belastungsstörung/Anpassungsstörung) sowie spezifische Phobien und reaktive depressive Verstimmungen

- chronifizierte Schmerzzustände (z. B. somatoforme Schmerzstörungen)

- latentes Rentenbegehren

Das Leistungsspektrum umfasst:

- Psychodiagnostik hinsichtlich psychischer Störungen nach dem Arbeitsunfall (einschließlich standardisierter psychologischer Testverfahren)
- supportive Psychotherapie zur psychischen Stabilisierung als präventive Maßnahme zur Vermeidung einer Chronifizierung von Beschwerden bzw. zur Therapiemotivation

- Krisenintervention bei akuter psychischer Dekompensation im Behandlungsverlauf

- multimodale psychotherapeutische Intervention unter Einbeziehung von Entspannungsverfahren, Biofeedbackmethoden sowie Gruppentherapien

- psychotherapeutische Mitbehandlung im Rahmen einer interdisziplinären Schmerztherapie (Vermittlung von Entspannungsverfahren und kognitiven Ablenkungsstrategien, Biofeedbackmethoden, imaginative Verfahren)

- Erarbeitung psychologischer Begutachtungen im Rahmen von Unfallzusammenhangsgutachten

- Möglichkeit der Abgrenzung prämorbider psychischer Erkrankungen von psychoreaktiven Störungen infolge des Unfalls als Entscheidungsgrundlage für eine optimale Heilverfahrenssteuerung

- ambulante psychotherapeutische Weiterbehandlung nach Abschluss der stationären Rehabilitation in unserer psychotraumatologischen Ambulanz

- Vermittlung wohnortnaher niedergelassener Psychotherapeuten bzw. spezialisierter psychotherapeutischer Kliniken nach entsprechender Indikationsstellung

\section{Physikalische und rehabilitative Medizin}

Unter Aufsicht eines Arztes für physikalische und rehabilitative Medizin arbeiten die Kernbereiche Physiotherapie, Ergotherapie und Sporttherapie am engsten mit den Patienten in der Handrehabilitation. Hierfür stehen speziell eingerichtete Räume einschließlich einer Sporthalle und 2 Schwimmbädern zur Verfügung. Das in der Handtherapie eingesetzte Team verfügt über eine mehrjährige Erfahrung mit diesen speziellen Krankheitsbildern und nimmt regelmäßig an den Teamsitzungen teil.

\section{Leistungsangebot der Physiotherapie für die Handtherapie.}

- Krankengymnastik oder Bewegungstherapie
- Krankengymnastik auf neurophysiologischen Grundlagen, wie PNF, Bobath, Vojta

- manuelle Therapie

- Krankengymnastik im Schlingentisch oder an diversen Kraftgeräten

- Atem-, Elektro-, Kryo- und Kaltlufttherapie

- Paraffinbad, speziell für Handverletzungen

Leistungsangebot der Bäderabteilung für die Handtherapie.

- klassische Massage

- manuelle Lymphdränage

- Unterwasserdruckstrahlmassage

- Reflexzonenmassage

- Ultraschall, Phonophorese

- Kurzwelle, Strahler, Laser, Elektrotherapie

- Fango-Paraffin-Packungen

- hydroelektrische sowie Vierzellenbäder

- $\mathrm{CO}_{2}$-Bäder

\section{Leistungsangebot der Ergotherapie für} die Handtherapie.

- motorisch-funktionelle Behandlung

- sensomotorisch-perzeptive Behandlung

- unterstützende thermische Behandlung

- berufsbezogenes Training im Werkstattbereich

- Austestung beruflicher Belastungsprofile

- Versorgung mit temporären thermoplastischen Schienen

- Versorgung mit Hilfsmitteln und Adaptationen für Gebrauchsgegenstände mit thermoplastischen und anderen entsprechenden Materialien

- ergotherapeutische Beratung des Betroffenen und seiner Angehörigen zur Wiedereingliederung in das soziale, berufliche und schulische Leben

- Austestung und Unterstützung bei verletzungsadaptierten Umbauten am Arbeitsplatz und in der Wohnung

- Training im Übungsauto

\section{Leistungsangebot der Sporttherapie für} die Handtherapie.

- medizinische Trainingstherapie

- Test und Training am BIODEX 3 - Isokinetik 
- Training im Bewegungsbecken; einzeln und in der Gruppe

- Kegeln, Bogenschießen

- Schwimmen

- Laufband- und Ergometertraining

- Sport, Sportspiele

- Herz-Kreislauf-Training

\section{Handchirurgisches Funktionslabor}

Es dient der standardisierten und computergestützten Befunddokumentation und der Erhebung weiterer Befunde. Das Leistungsspektrum umfasst:

- computergestützte Handfunktionsmessung für Kraft und Beweglichkeit (- Abb. 1)

- Messung von Gewebedurchblutung

- Videothermographie in Echtzeit in Ruhe und unter Belastung zur Bestimmung von Durchblutungsstörungen

- Kapillarperfusionsmessungen in Echtzeit zur Bestimmung von Durchblutungsstörungen

- Foto- und Videodokumentation

- computergestützte Analyse der Auswirkungen von Muskelverspannungen und Fehlbelastungen der oberen Extremitäten auf den Schultergürtel und die Auswirkungen der Therapie (im Aufbau)

\section{Berufshilfe}

Sie stellt über den gesamten Verlauf den engen Kontakt zur betreuenden BG und dem Betrieb her. Sie berät den Verletzten über seine Möglichkeiten, organisiert die Wiederaufnahme der Arbeit und eine evtl. Belastungserprobung. Bei Einleitung berufsfördernder Maßnahmen ist sie die zentrale Kontaktstelle für den Kostenträger. Angeboten werden:

- Einleitung der beruflichen Rehabilitation

- Beratung in allen sozialrechtlichen Angelegenheiten

- Brückenfunktion zum Sachbearbeiter und Berufshelfer der betreuenden BG

- Beratung bei Problemen im beruflichen und persönlichen Umfeld

- Vermittlung ambulanter Hilfen

- Unterstützung bei der Kraftfahrzeughilfe

- Beratung und Unterstützung bei Umbauten am Arbeitsplatz und in der Wohnung

Jamar (TM)

Grifftest:

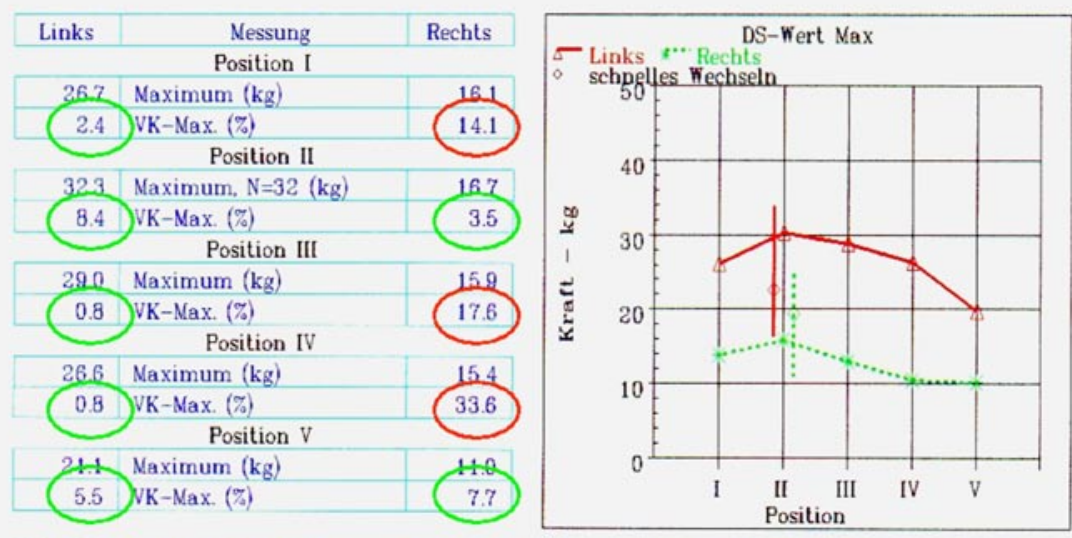

Links: Die maximale Griffkraft beim Test "schnelles alternierendes Wechseln" war $5 \%$ mehr als Die maximale Leistung beim Funf-Stufentest.

Rechts: Die maximale Griffkraft beim. Test "schnelles alternierendes Wechseln" war $50 \%$ mehr als Die maximale Leistung beim Funf-Stufentest.

Abb. $1 \Delta$ Messprotokoll einer computergestützten Handfunktionsmessung im Funktionslabor, rot umkreiste Werte hohe Streubreite der Einzelversuche bei wiederholten Messungen als Hinweis auf den Versuch des Verletzten, seine Leistung schlechter darzustellen, als sie in Wahrheit ist; beweisend in diesem Fall: Leistung bei schnellen Griffwechseln an der verletzten Hand um $\mathbf{5 0 \%}$ höher als bei Messserien an nur jeweils einer Hand (unterstrichene Textpassage am Boden des Bildes), Ursache: Kontrollverlust, welcher bei schnellen Wechseln erreicht wird

- Vorbereitung und Organisation des nahtlosen Übergangs aus der handtherapeutischen Rehabilitation in die Arbeits-Belastungs-Erprobung (ABE)

- Kontrolle während der Belastungserprobung

\section{Orthopädietechnik}

- Ausmessen, Liefern und Anpassung von Bandagen, Schienen

- Versorgung mit Hilfsmitteln

- Fertigung von Arbeitshandschuhen nach $\mathrm{Ma}$

- Lieferung, Anpassen und Ändern von Prothesen

\section{Pflege der handchirurgischen Station}

Sie versorgt medizinisch alle Verletzten, welche aufgrund ihrer Einschränkungen oder begleitender Verletzungen einer Pflege und Versorgung bedürfen. Für alle anderen Verletzten, welche in Appartements ohne Stationspflege untergebracht sind, ist der Stationsstützpunkt die organisatorische Anlaufstelle. Hier werden alle Krankenunterlagen geführt, alle Leistungen beauftragt und EDV-technisch abgewickelt. Alle Befunde laufen hier zusammen und werden den beteiligten Ärzten zugeleitet. Die Patienten in der handtherapeutischen Rehabilitation finden hier für ihre Fragen ihren Ansprechpartner.

\section{Funktionsablauf}

\section{Zuweisung}

Ob eine spezielle handtherapeutische Rehabilitation zweckmäßig ist, entscheidet sich im BG-lichen Heilverfahren auf verschiedenen Wegen.

1. Verletzte mit schwer wiegenden Verletzungen und Funktionsstörungen befinden sich in der Behandlung der Klinik. Die handtherapeutische Rehabilitation ist Teil des gesamten Rehabilitationsplans.

2. Verletzte befinden sich anderweitig in Behandlung. Die behandelnden Ärzte sehen die Notwendigkeit einer speziellen handtherapeutischen Rehabilitation.

Es erfolgen nach Abstimmung mit dem Kostenträger Vorstellung als Heilverfahrenskontrolle (HVK) und bei Bestätigung des Konzepts kurzfristige Aufnahme. 
3. Der das Heilverfahren überwachende Sachbearbeiter sieht aus dem Berichtswesen und/oder der Dauer des Heilverfahrens die Notwendigkeit einer HVK. Er veranlasst diese und erhält eine Stellungnahme, die die weiteren therapeutischen Optionen darlegt. Sofern darin eine handtherapeutische Rehabilitation vorgeschlagen und entsprechende Behandlungsziele benannt werden, prüft er die Genehmigung. Nach deren Erteilung wird der/die Verletzte aufgenommen.

4. Der Verletzte selbst wendet sich an seinen behandelnden Arzt oder an den Sachbearbeiter mit dem Wunsch nach Intensivierung der Rehabilitation. Dann prüfen diese die Situation und verfahren nach den Punkten 2 oder 3.

Der Verletzte wird im Rahmen der vor der Aufnahme stattfindenden HVK auf seine Rehabilitationstauglichkeitund das Rehabilitationspotenzial geprüft. Es wird außerdem eruiert, ob vorher operative Maßnahmen erforderlich sind oder sich nach der Rehabilitation noch anschließen, welche die Handtherapie als Voraussetzung brauchen.

Vom Handchirurgen wird eine ausführliche Stellungnahme im Rahmen der HVK abgegeben, welche gleichzeitig Basis der ersten Aktivitäten im Rehabilitationsteam ist.

Sofern der Rahmen der ambulanten Heilverfahrenskontrolle nicht ausreicht, das Rehabilitationspotenzial ausreichend abzuschätzen, wird mit den Ressourcen des handtherapeutischen Rehabilitationsteams eine 2-tägige Austestung durchgeführt, an deren Ende eine entsprechend detaillierte Stellungnahme erfolgt.

\section{Abschätzung des Rehabilitationspotenzials}

Am 1. Tag werden in jedem Funktionsbereich eine Befunderhebung durchgeführt und

1. noch offene diagnostische Fragen benannt,

2. die wesentlichen Funktionseinschränkungen mit ihren Ursachen festgehalten,

3. die Belastbarkeit des/der Verletzten eingestuft,
4. die Therapieziele für den Bereich formuliert.

Am 2. Tag erfolgt die Abstimmung der einzelnen Bereiche über die handchirurgischen Ärzte unter Formulierung eines Therapiekonzepts und Definition der Therapieziele. Auch noch notwendige erweiterte Diagnostik wird durchgeführt.

Die Therapieansätze werden in einer Teamsitzung mit allen Beteiligten koordiniert. Mit dem/der Verletzten werden der Therapieplan durchgesprochen und die Rehabilitationsziele festgelegt.

So weit die Aufnahme zur Therapie erfolgte, sind die diagnostischen Schritte schon von den vorher definierten Therapieabschnitten begleitet. Erfolgte die Aufnahme allein zur Abschätzung des Rehabilitationspotenzials, wird dem Kostenträger berichtet und abhängig von medizinischen Vorschlag und der Entscheidung des Kostenträgers weiter verfahren.

\section{Rehabilitationsverlauf}

Die Überprüfung und ggf. Neubestimmung der koordinierten Therapie erfolgen in den Teamsitzungen.

Es werden von Beginn an 2-mal wöchentlich standardisiert Messwerte aller für den Verlauf wesentlichen Funktionen erhoben. Diese gehen in die Teamsitzungen neben den Befunden der einzelnen Bereiche ein.

So weit sich Gesichtspunkte ergeben, welche Veränderungen der Therapie bedingen, werden diese in der Teamsitzung beschlossen und alle anderen Bereiche daran angepasst. Sofern innerhalb der Sitzungsintervalle Veränderungen nötig sind, werden sie in direkter Abstimmung aller betroffenen Bereiche über den Handchirurgen koordiniert.

Der Zeitbedarf für eine handtherapeutische Rehabilitation beträgt meist 3 Wochen. Sofern eine Verlängerung erforderlich scheint, wird sie mit Begründung auf Basis des bisherigen Therapieverlaufs beim Kostenträger beantragt.

\section{Abschluss}

Im Rehabilitationsverlauf wird frühzeitig die Basis für die berufliche Wiedereingliederung mit dem Verletzten abgestimmt.
Ziel ist, mit Abschluss nahtlos in die berufliche Belastungserprobung überzugehen. Ausgenommen sind nur Rehabilitationsabschnitte, welche bei schweren Verletzungen im Verlauf eingeschaltet werden, ohne dass das Ziel schon im Abschluss besteht.

Sofern eine MdE verbleibt, wird dies dem Kostenträger frühzeitig mitgeteilt. Wenn gewünscht, kann der Abschluss mit einer Begutachtung erfolgen, sodass hier eine zügige Bearbeitung und ein erneuter Arbeitsausfall nach der Wiedereingliederung vermieden werden können.

Im Verlauf einer sich anschließenden beruflichen Belastungserprobung werden die Zwischenkontrollen weiterhin über das Rehabilitationsteam organisiert und damit sichergestellt, dass weiter eine straffe Führung des Heilverfahrens erfolgt.

\section{Dokumentation und Berichtswesen}

Alle technischen Messverfahren sind computergestützt, sodass reproduzierbare Messwerte über den gesamten Verlauf dokumentiert werden. Damit ergeben sich verlässliche Aussagen über Behandlungsfortschritt und -ergebnis.

Alle Verläufe und Ergebnisse der einzelnen Bereiche werden dokumentiert und im Abschlussbericht an den Kostenträger zusammengefasst. Dem Bericht werden Anlagen der Messergebnisse und Fotodokumentation beigefügt. Mitbehandelnde oder vorher in die Behandlung einbezogene Ärzte und Kliniken werden parallel informiert. So ergibt sich ein nachvollziehbares Bild des Behandlungsablaufs, der erreichten Ergebnisse und des Leistungsvermögens des Verletzten.

\section{Korrespondierender Autor PD Dr. M. Steen}

Klinik für Plastische und Handchirurgie, Brandverletztenzentrum,

BG-Kliniken Bergmannstrost, Merseburger Straße 165, 06112 Halle, Saale E-Mail:

plastische-chirurgie@bergmannstrost.com

Interessenkonflikt: Der korrespondierende Autor versichert, dass keine Verbindungen mit einer Firma, deren Produkt in dem Artikel genannt ist, oder einer Firma, die ein Konkurrenzprodukt vertreibt, bestehen. 